\title{
Circumferential dural resection technique and reconstruction for the removal of giant calcified transdural herniated thoracic dises
}

\author{
Corey T. Walker, MD, ${ }^{1}$ M. Yashar S. Kalani, MD, PhD, ${ }^{1}$ Mark E. Oppenlander, MD, ${ }^{1}$ \\ Jakub Godzik, MD, ${ }^{1}$ Nikolay L. Martirosyan, MD, ${ }^{1}$ Robert J. Standerfer, MD, ${ }^{2}$ and \\ Nicholas Theodore, MD'
}

Departments of ${ }^{1}$ Neurosurgery and ${ }^{2}$ Surgery, Barrow Neurological Institute, St. Joseph's Hospital and Medical Center, Phoenix, Arizona

OBJECTIVE The authors report a novel paradigm for resection of the disc or dural complex to treat giant calcified transdural herniated thoracic discs, and they describe a technique for the repair of dural defects. These herniated thoracic discs are uncommon, complicated lesions that often require a multidisciplinary team for effective treatment. The intradural component must be removed to effectively decompress the spinal cord. The opening of the friable dura mater, which frequently adheres to the extradural component of the disc, can result in large defects and difficult-to-manage CSF leaks.

METHODS The authors performed a retrospective study of the technique and outcomes in patients with a transdural herniated disc treated at St. Joseph's Hospital and Medical Center within a 4-year period between 2012 and 2015.

RESULTS During the study period, 7 patients (mean age 56.1 years) presented to the department of neurosurgery with clinical symptoms consistent with myeloradiculopathy. In all cases, 2-level corpectomies of the involved levels were combined with circumferential resection of the dura and complete decompression of the spinal cord. The dural defect was repaired with an onlay dural patch, and a large piece of AlloDerm (LifeCell Corp) graft was sewn to close the pleural defect. Every patient had a perioperative lumbar drain placed for CSF diversion. No patient suffered neurological decline related to the surgery, and 3 patients experienced clinically significant improvement in function. Two patients developed an early postoperative CSF leak that required operative revision to oversew the defects.

CONCLUSIONS This novel technique for decompression of the spinal cord by dural resection for the removal of giant calcified transdural herniated thoracic discs is safe and results in excellent decompression of the spinal cord. The technique becomes necessary when primary repair of the dura is not possible, and it can be used in cases in which the resection of pathology includes the dura.

https://thejns.org/doi/abs/10.3171/2017.5.SPINE161285

KEY WORDS calcified; disc; herniated; surgical technique; thoracic; thoracotomy; transdural

I NTERVERTEBRAL disc herniation occurs when a rupture of the fibrous tissue surrounding the nucleus pulposus allows the nucleus of the compromised disc to invade the epidural space of the spinal canal and, in some cases, to compress the spinal cord. ${ }^{1}$ Although symptomatic herniated thoracic discs are rare (only 1 in 1,000,000 persons), the sequelae of the herniation can be devastating. ' When more than $40 \%$ of the spinal canal is occluded by such a herniation, the rupture is considered "giant," will often adhere to the dura mater, eventually become calcified, and, in some cases, traverse the dural leaflets to impinge on the spinal cord. This giant transdural prolapse invading the plane between the dura and the calcified disc ${ }^{9}$ is associated with substantial difficulty during resection because it hinders their safe separation. ${ }^{10}$

During surgery for a transdural herniated thoracic disc, opening the dura to resect the intradural component is frequently necessary to decompress the spinal cord effectively. However, in such cases, opening the dura can result in CSF leakage or a CSF-pleural fistula. These complications have driven some surgeons to leave small fragments of adherent disc attached to the dura to avoid CSF leakage.

Although the resection of a transdural herniated thoracic disc can enable primary dural repair, we have encountered a subset of cases in which the dura cannot be repaired primarily; in these cases the thoracic dura cannot be sewn 
because of the friable nature of the dural leaflets after resection of the disc or because of the large size of the dural defect after disc removal. Therefore, we developed a technique for resecting transdural herniated thoracic discs that involves resection of the calcified disc and the surrounding dural complex together. That is, we circumferentially incise the dura around the borders of the calcified herniated disc. This circumferential dural resection technique is combined with a novel method by which CSF leaks can be prevented in patients with this difficult condition. Herein, we describe this new technique and report the surgical outcomes of a small series of patients treated for a giant calcified transdural herniated thoracic disc.

\section{Methods \\ Patients}

Over a 4-year period between 2012 and 2015, a total of 7 patients ( 6 female, 1 male) with a mean age of 56.1 years presented with clinical symptoms consistent with myeloradiculopathy. MRI and CT findings of a giant calcified transdural herniated thoracic disc confirmed the diagnosis and fit each patient's clinical symptoms. Disc herniation occurred in spinal levels T6-11 in each of the 7 patients. No patient with multiple thoracic discs that required surgery was included. Each patient who underwent surgery was healthy enough for surgical treatment and was cleared medically for surgery by either a hospitalist or a critical care intensivist familiar with the patient's condition and comorbidities. Intraoperative neuromonitoring was conducted in all 7 cases. According to our review of the operative reports, no loss of neuromonitoring responses was recorded. The preoperative and postoperative Frankel grade $^{14}$ of each patient was recorded, and all the patients either remained at their baseline neurological status or improved after surgery.

\section{Surgical Technique}

The surgical technique for each of the 7 patients consisted of open thoracotomy performed by members of our cardiothoracic team. The level of interest is confirmed intraoperatively, either with a radiolucent marker that has been placed previously under CT guidance at the pedicle of the level of interest ${ }^{11,19}$ or by carefully counting ribs. On occasion, the calcified disc is apparent on intraoperative radiographs. In patients with a giant calcified transdural herniated thoracic disc, we prefer to perform corpectomies of the 2 involved vertebrae to provide wide exposure for visualization and manipulation of the disc.

After the corpectomies are performed, the fragment of disc is visualized and inspected to reveal the transdural nature of the disc. Thus, the dura can be cut sharply and circumferentially around the protruding disc. All dissecting motions of the disc are performed with force away from the spinal cord and into the corpectomy defects. This process enables the removal of the disc, dura, or osteophyte complex with minimal or no manipulation of the spinal cord. After complete resection of the compressive lesion, the spinal cord is visualized. The dura is covered with an onlay dural substitute (DuraGen [Integra LifeSciences Corp.]) and Tisseel fibrin sealant (Baxter Healthcare
Corp.). Then, the spine is stabilized with either a strut allograft or an expandable cage, followed by plate and screw fixation and autograft or allograft fusion. After the fusion is performed, a large piece of AlloDerm graft (LifeCell Corp.) is sewn to the pleura over the site of the defect. This pleural patch graft covers the dural defect and the fusion construct in a watertight fashion. Mechanical pleurodesis is performed using electrocautery (Bovie Medical Corp.), and chemical pleurodesis is performed using tetracycline. A chest tube is placed before final closure, and then a lumbar drain is placed for CSF diversion.

For the first 48-72 hours, the patient is kept intubated to maintain positive-pressure ventilation. The chest tube and lumbar drain are also maintained in place. The chest tube is set to water seal only. The lumbar drain is placed at ear level to promote drainage of CSF through the lumbar drain and away from the thoracic dural defect. We believe that these procedures help prevent the formation of a CSFpleural fistula and allow the site of repair to heal. We remove the chest tube when the output is less than $30 \mathrm{ml}$ over 8 hours. The lumbar drain is removed only after the chest tube has been removed. The patient then can be mobilized.

\section{Results}

The characteristics of the 7 patients in this study are summarized in Table 1. All the patients presented with thoracic myelopathy and were found to have a giant calcified transdural herniated thoracic disc. All the patients underwent at least 6 months of postsurgical follow-up. Despite careful dural closure, 2 patients experienced a postoperative CSF leak that required a return to the operating room to oversew the leak. None of the patients had a persistent CSF-pleural fistula at the last follow-up visit.

For unknown reasons, 1 female patient experienced posterior reversible encephalopathy syndrome after surgery, which resulted in impaired neurological function. This patient had a history of low-grade glioma and experienced altered mental status on Day 4 after surgery. We believe that this outcome was unrelated to the surgical intervention but instead might have resulted from iatrogenic manipulation of her blood pressure during hospitalization. Although her mental status was affected by the encephalopathy, her physical strength continued to improve in the following weeks.

\section{Illustrative Case}

A healthy 57-year-old woman (Case 1) presented with signs and symptoms consistent with thoracic myeloradiculopathy. She had a months-long history of progressive radicular pain in the thoracic region that left her unable to walk. Motor examination of the patient's left side revealed proximal (Grade 2/5) and distal (Grade 3/5) weaknesses. She had numbness and a sensory level to approximately T-6. T2-weighted MR images revealed severe thoracic spondylosis and evidence of ossification of the posterior longitudinal ligament at multiple levels in the thoracic spine, notably T2-7 (Fig. 1A and B). CT images revealed a giant calcified transdural herniated thoracic disc at T6-7 that resulted in a myelographic block and cord compression (Fig. 1C and D). We believed that the patient would 
TABLE 1. Patient characteristics and outcomes

\begin{tabular}{|c|c|c|c|c|c|c|}
\hline \multirow{2}{*}{$\begin{array}{l}\text { Case } \\
\text { No. }\end{array}$} & \multirow{2}{*}{$\begin{array}{l}\text { Age (yrs) } \\
\text { Sex }\end{array}$} & \multirow{2}{*}{$\begin{array}{c}\text { Level of Disc } \\
\text { Herniation }\end{array}$} & \multirow[b]{2}{*}{ Presenting Symptom(s) } & \multicolumn{2}{|c|}{ Frankel Grade* } & \multirow{2}{*}{$\begin{array}{l}\text { CSF } \\
\text { Leak }\end{array}$} \\
\hline & & & & Preop & Postop & \\
\hline 1 & $57, \mathrm{~F}$ & T6-7 & Numbness below T-6; inability to ambulate & C & C & No \\
\hline 2 & $61, \mathrm{~F}$ & T10-11 & Progressive paraparesis & C & $\mathrm{D}$ & Yes \\
\hline 3 & $54, \mathrm{~F}$ & T10-11 & Severe pain on standing; pain radiating to groin & $\mathrm{D}$ & $E$ & No \\
\hline 4 & $59, \mathrm{~F}$ & T10-11 & Lower-extremity paresthesia & $\mathrm{D}$ & $E$ & No \\
\hline 5 & $41, \mathrm{~F}$ & T8-9 & Severe lower-extremity paraparesis; previous failed posterior thoracic exploration & B & C & No \\
\hline 6 & $67, \mathrm{M}$ & T7-8 & Lower-extremity weakness \& back pain; previous failed thoracic laminectomy & C & $\mathrm{D}$ & No \\
\hline 7 & $54, \mathrm{~F}$ & $\mathrm{~T} 7-8$ & Lower-extremity weakness \& numbness; falls & $\mathrm{C}$ & $\mathrm{D} \dagger$ & Yes \\
\hline
\end{tabular}

* Frankel grades are defined as follows: $A=$ complete interruption of all sensation and motor function; $B=$ incomplete interruption, some sensation but no motor function; $C=$ incomplete interruption, demonstrable voluntary motor function but at a minimal level, serving no useful purpose; $D=$ incomplete interruption, some voluntary motor function that is useful to the patient; $E$ = recovery to normal functioning attained.

$\dagger$ Complicated by posterior reversible encephalopathy syndrome. This patient had a history of low-grade glioma.

benefit from T-6 and T-7 corpectomies with resection of the giant calcified transdural herniated thoracic disc. Given the friable nature of the adjacent dura, we performed sharp dural resection around the lesion to deliver the disc en bloc (Fig. 1E), and a dural graft was used to create a watertight closure of the defect. Instrumentation and fusion were performed with an expandable cage in the corpectomy defects, autograft in the cage, and an anterolateral plate and screws spanning T5-8 (Fig. 1F). A pleural patch graft (Fig. 1G) was applied, followed by mechanical and chemical pleurodesis, and a chest tube and a lumbar drain were placed.

The initial postoperative examination of the patient found her remaining at her baseline neurological status. She remained intubated with positive-pressure ventilation for 48 hours. The chest tube was kept in place until the output was less than $30 \mathrm{ml}$ every 8 hours, and the lumbar drain was kept in place until the chest tube was removed. On Day 9, the patient remained at her neurological baseline, and no evidence of CSF leakage was found. She was discharged to a rehabilitation facility after removal of the lumbar drain. At the 6-month follow-up visit, the patient was living at home with her family. Her wound was well healed, and she exhibited no evidence of CSF leakage. She was able to ambulate for 15 minutes twice per day and could care for herself, but she needed assistance with rising from a sitting position and with showering.

\section{Discussion}

Herniated thoracic discs represent a small portion of all vertebral disc herniations $(0.15 \%-0.5 \%$ of all disc disease hospitalizations). ${ }^{6}$ However, their presentation is often debilitating and must be treated urgently and appropriately, especially in patients with a giant calcified transdural herniated thoracic disc and progressive symptoms. Many experts recommend an anterior approach to best address the compressive pathology while minimizing injury to the spinal cord. ${ }^{7,10}$ Posterolateral approaches are excellent options for lateralizing discs, but they provide insufficient visualization of the ventral dural surface; therefore, they increase the risk for spinal cord injury and incomplete disc removal in patients with midline herniation. ${ }^{8}$
Of the anterior approaches, open thoracotomy confers substantial advantages for the treatment of giant calcified transdural herniated thoracic discs, namely, direct access to the disc and visualization of the compression without the need for spinal cord manipulation..$^{16}$ Although thoracoscopic surgery can be considered for less-invasive access to the ventral corridor, this approach is complicated by the associated high rate of dural tears and conversion to an open approach, especially when large calcified disks with dural invasion are involved. ${ }^{9,10}$ Giant calcified herniated thoracic discs that are transdural in nature require the dura to be opened to enable complete resection of the pathology. In such cases, open thoracotomy provides the surgeon the greatest opportunity to achieve watertight dural closure.?

Resection of a subset of giant calcified transdural herniated thoracic discs might result in a dural defect that cannot be closed, primarily because of the large nature of the defect and the friable nature of the remaining dura. Several groups have described their experience with anterior approaches to giant herniated thoracic discs, but the surgical literature does not adequately describe how to manage patients with such a complex condition. ${ }^{2,3,17,20} \mathrm{We}$ believe that such patients initially require circumferential resection of the disc and surrounding dura and subsequent measures to prevent CSF-pleural fistulas. In this report, we have described our novel technique for resecting the ossified transdural disc and the surrounding dura together. This circumferential dural resection technique was undertaken after intraoperative recognition that the disc was transdural in nature and that no clear plane existed between the surrounding friable dura and the giant herniated disc. We created large corpectomy defects into which the disc-dural complex could be delivered safely, away from the already compromised spinal cord. With this technique, all 7 patients in our series underwent complete resection of their giant calcified transdural herniated thoracic disc without neurological compromise (Table 1).

In addition, the technique involves multiple maneuvers to prevent postoperative CSF egress from the resultant dural defect. A dural substitute onlay is placed directly over the defect before instrumentation and fusion are performed. Then, a watertight AlloDerm patch graft is sewn 

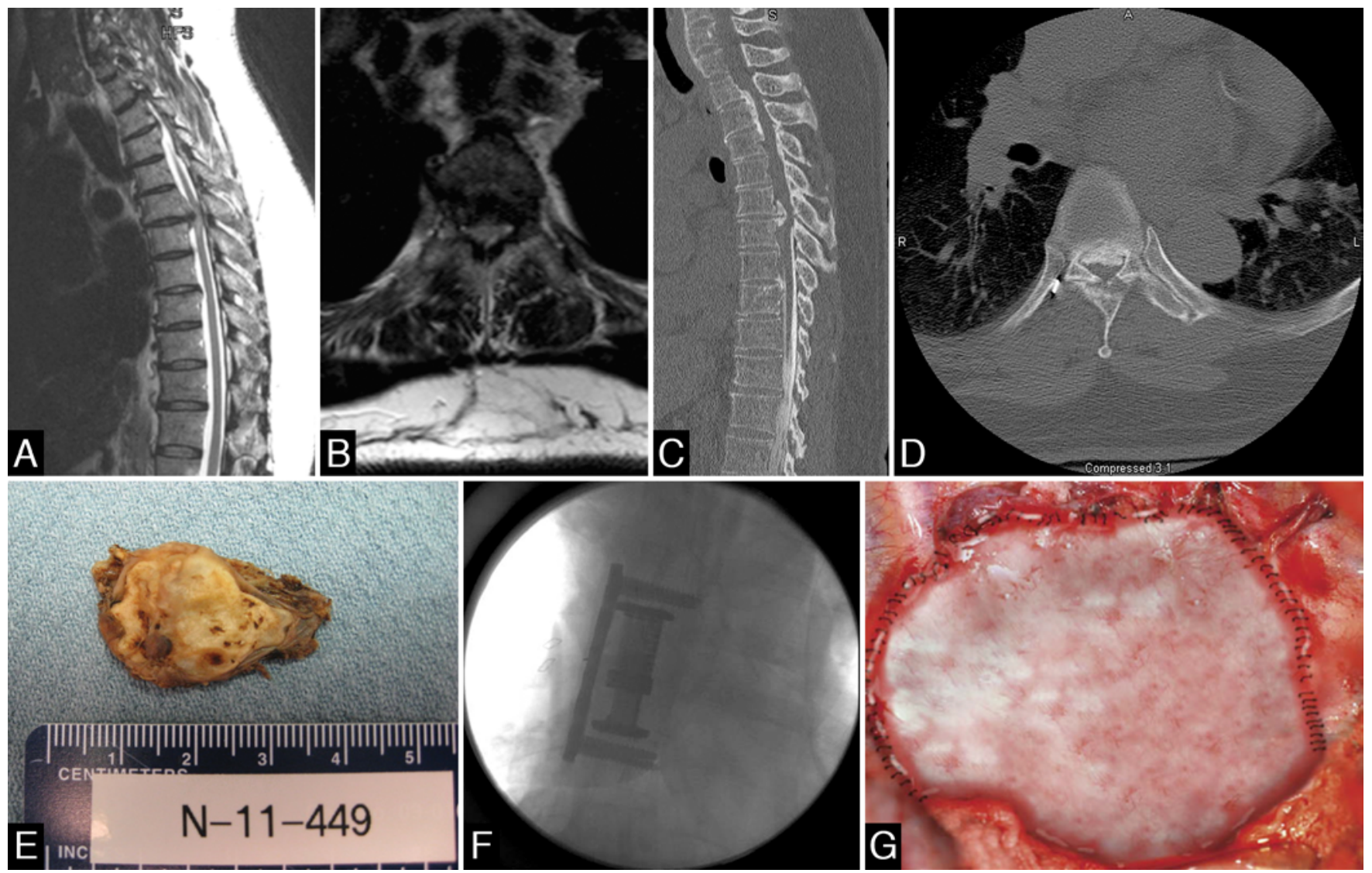

FIG. 1. A and B: Sagittal (A) and axial (B) T2-weighted MR images reveal an anterior compressive pathology effacing the spinal cord at T6-7 and smaller areas of disc herniation at other thoracic levels. C and D: Sagittal (C) and axial (D) CT images reveal a disc or osteophyte complex obliterating more than $50 \%$ of the spinal canal at the level of T6-7, with myelographic block. A giant calcified transdural disc was found intraoperatively to be compressing the spinal cord. E: The disc or osteophyte complex was resected circumferentially and removed. F: After the fusion was completed, a large piece of AlloDerm was sewn to the pleura to cover the site of the dural defect and to prevent egress of CSF into the pleural cavity. G: Intraoperative photograph of the graftsewing process. Figure is available in color online only.

to the pleura over the fusion construct. These initial maneuvers provide the primary barrier for CSF egress into the pleural space, but several adjuvant safety measures are also instituted. First, placement of a chest tube and lumbar drain helps decrease the pressure within each respective compartment and hastens the resolution of undesired flow from the thecal space. In multiple studies of lumbar drain use, this approach has been shown to decrease the incidence of CSF fistulas after anterior repair of leaks acquired during decompression for ossification of the posterior longitudinal ligament. ${ }^{15}$ It is important to note that placing a chest tube on wall suction can have devastating consequences and can actually instigate a CSF leak, which places the patient at risk for brain herniation and pneumocephalus if sufficient inadvertent CSF is drained under negative pressure. ${ }^{5,13}$ Second, we believe that a combination of mechanical and chemical pleurodesis further decreases the risk of CSF leak or fistula formation within the pleural space. Mechanical and chemical pleurodesis has been used successfully by cardiothoracic surgeons to eliminate pleuroperitoneal connections, as observed in the circumstance of hydrothorax secondary to elevated intraabdominal fluid pressure from ascites. ${ }^{4}$ Therefore, it might help promote desired fibrogenesis in susceptible areas of the pleura because the pathophysiologies of these 2 processes are similar. We did not identify any complications associated with the use of chemical pleurodesis with tetracycline. Third, we favor keeping patients intubated for the first 48 hours after surgery. Doing so enables us to maintain positive-pressure ventilation and thereby alter the pressure gradient and increase resistance to CSF flow into the intrathoracic space.

With the use of these measures, no cases of ongoing CSF-pleural fistulas occurred in our series. In 2 patients, a CSF leak that required reopening to oversew the defects occurred early in the postoperative course. Both patients went on to do well and showed no further evidence of CSF leakage or long-term sequelae. Although we recognize that the development of this complication in $2(29 \%)$ of the 7 patients represents a substantial percentage, this incidence is within the expected range reported by other groups performing anterior thoracic decompression surgeries. Little is known about the exact rates of CSF-pleural fistulation after transpleural thoracic resection of transdural disc herniations, given the rare nature of this pathology and the diversity of surgical approaches. That said, Hu et al. ${ }^{12}$ 
recently reported on a series of thoracic decompression surgeries in which they found a $21 \%$ rate of postoperative CSF leakage after 39 anterior approaches. Because this statistic is representative of all thoracic disc herniations, including small, noncalcified, and nontransdural herniations, our rate is actually quite favorable. In a more congruous study, Roelz et al. ${ }^{18}$ noted that 1 of 6 patients who underwent a minitransthoracic approach for transdural giant herniated thoracic disc resection experienced CSFpleural fistula complications. Thus, we believe that such complications are unavoidable, even after multiple preventive measures (e.g., watertight dural closure, fibrin glue, pleural patch, lumbar drain, pleurodesis) are undertaken to help deter this undesirable connection, because a large dural opening is required.

Another patient experienced postoperative altered mental status and symptoms consistent with posterior reversible encephalopathy syndrome on Day 4 after surgery. We believe that this outcome was unrelated to our intervention but, instead, might reflect iatrogenic manipulation of the patient's blood pressure during hospitalization. Because her strength improved despite her encephalopathy, we deemed her surgery to be successful. Given the difficult nature of the operation to treat giant calcified transdural herniated thoracic discs safely and effectively, these complications are acceptable, and the described technique for resecting such lesions seems safe. In addition, these adverse outcomes seem preferable to incomplete dural resection or further neurological deficits resulting from spinal cord injury, which can be encountered with alternative approaches.

It is also important to note that nonneurological complications, including (but not limited to) ventilator dependence, pneumonia, venous thromboembolism, and genitourinary infection, are common after such complex transthoracic procedures. A limitation of this study is that its retrospective nature prevented us from appropriately considering these outcomes in relation to the burden inherent to this procedure. Preoperative counseling and weighing of the risks are important components of surgical planning in this patient population. Moreover, given that this was a retrospective cohort study, we were unable to compare the outcomes of our 7 patients with those of a control group to assess relative outcomes for this procedure compared with those of other surgical approaches or technical strategies. Continued efforts are necessary to improve existing techniques to manage this difficult disease process.

\section{Conclusions}

Giant calcified transdural herniated thoracic discs represent a difficult-to-treat condition complicated by burdens such as the need for ventral thoracic surgical access, substantial disc adherence to the dura, calcification, and a high risk of neurological compromise. Wide circumferential resection of the herniated disc and removal of compressive pathology allow for an excellent neurological outcome in certain patients with such pathology. When resection of a disc and dura is necessary, our technique enables repair of the dural defect, prevents CSF-pleural fistulation, and is associated with minimal complications. Special attention to adjunct measures might help suppress CSF egress while definitive closure of the dural graft and pleura occurs in the immediate postoperative period.

\section{References}

1. Arce CA, Dohrmann GJ: Thoracic disc herniation. Improved diagnosis with computed tomographic scanning and a review of the literature. Surg Neurol 23:356-361, 1985

2. Ayhan S, Nelson C, Gok B, Petteys RJ, Wolinsky JP, Witham TF, et al: Transthoracic surgical treatment for centrally located thoracic disc herniations presenting with myelopathy: a 5-year institutional experience. J Spinal Disord Tech 23:79-88, 2010

3. Barbanera A, Serchi E, Fiorenza V, Nina P, Andreoli A: Giant calcified thoracic herniated disc: considerations aiming a proper surgical strategy. J Neurosurg Sci 53:19-26, 2009

4. Boin IF, Silva AM, Leonardi LS: Chemical pleurodesis for hepatic hydrothorax. Arq Gastroenterol 38:125-128, 2001

5. Chaudhry MS: Intracranial hypotension caused by duralpleural fistula. J Neuroimaging 22:208-209, 2012

6. Delfini R, Di Lorenzo N, Ciappetta P, Bristot R, Cantore G: Surgical treatment of thoracic disc herniation: a reappraisal of Larson's lateral extracavitary approach. Surg Neurol 45:517-523, 1996

7. Dickman CA, Mican CA: Multilevel anterior thoracic discectomies and anterior interbody fusion using a microsurgical thoracoscopic approach. Case report. J Neurosurg 84:104109, 1996

8. Dickman CA, Rosenthal D, Regan JJ: Reoperation for herniated thoracic discs. J Neurosurg 91 (2 Suppl):157-162, 1999

9. Gille O, Soderlund C, Razafimahandri HJ, Mangione P, Vital JM: Analysis of hard thoracic herniated discs: review of 18 cases operated by thoracoscopy. Eur Spine J 15:537-542, 2006

10. Hott JS, Feiz-Erfan I, Kenny K, Dickman CA: Surgical management of giant herniated thoracic discs: analysis of 20 cases. J Neurosurg Spine 3:191-197, 2005

11. Hsu W, Sciubba DM, Sasson AD, Khavkin Y, Wolinsky JP, Gailloud P, et al: Intraoperative localization of thoracic spine level with preoperative percutaneous placement of intravertebral polymethylmethacrylate. J Spinal Disord Tech 21:72-75, 2008

12. Hu P, Yu M, Liu X, Liu Z, Jiang L, Wei F, et al: Cerebrospinal fluid leakage after surgeries on the thoracic spine: a review of 362 cases. Asian Spine J 10:472-479, 2016

13. Kalani MY, Filippidis A, Martirosyan NL, Theodore N: Cerebral herniation as a complication of chest tube drainage of cerebrospinal fluid after injury to the spine. World Neurosurg 79:798.E17-798.E19, 2013

14. Kirshblum SC, Burns SP, Biering-Sorenson F, Donovan W, Graves DE, Jha A, et al: International standards for neurological classification of spinal cord injury (Revised 2011). J Spinal Cord Med 34:535-546, 2011

15. Mazur M, Jost GF, Schmidt MH, Bisson EF: Management of cerebrospinal fluid leaks after anterior decompression for ossification of the posterior longitudinal ligament: a review of the literature. Neurosurg Focus 30(3):E13, 2011

16. Oppenlander ME, Clark JC, Kalyvas J, Dickman CA: Surgical management and clinical outcomes of multiple-level symptomatic herniated thoracic discs. J Neurosurg Spine 19:774-783, 2013

17. Quraishi NA, Khurana A, Tsegaye MM, Boszczyk BM, Mehdian SM: Calcified giant thoracic disc herniations: considerations and treatment strategies. Eur Spine J 23 (Suppl 1):S76-S83, 2014

18. Roelz R, Scholz C, Klingler JH, Scheiwe C, Sircar R, Hubbe $\mathrm{U}$ : Giant central thoracic disc herniations: surgical outcome 
in 17 consecutive patients treated by mini-thoracotomy. Eur Spine J 25:1443-1451, 2016

19. Upadhyaya CD, Wu JC, Chin CT, Balamurali G, Mummaneni PV: Avoidance of wrong-level thoracic spine surgery: intraoperative localization with preoperative percutaneous fiducial screw placement. J Neurosurg Spine 16:280-284, 2012

20. Zhao Y, Wang Y, Xiao S, Zhang Y, Liu Z, Liu B: Transthoracic approach for the treatment of calcified giant herniated thoracic discs. Eur Spine J 22:2466-2473, 2013

\section{Disclosures}

The authors report no conflict of interest concerning the materials or methods used in this study or the findings specified in this paper.

\section{Author Contributions}

Conception and design: Theodore. Acquisition of data: Theodore, Walker, Kalani. Analysis and interpretation of data: Walker, Kalani. Drafting the article: Walker, Kalani. Critically revising the article: Oppenlander, Godzik, Martirosyan. Reviewed submitted version of manuscript: Theodore. Statistical analysis: Walker, Kalani. Administrative/technical/material support: Standerfer. Study supervision: Theodore.

\section{Correspondence}

Nicholas Theodore, c/o Neuroscience Publications, Barrow Neurological Institute, St. Joseph's Hospital and Medical Center, 350 W Thomas Rd., Phoenix, AZ 85013. email: neuropub@ dignityhealth.org. 